\title{
ANÁLISE PARAMÉTRICA DO ESCOAMENTO DE UM FLUIDO DEVIDO À CONVECÇÃO NATURAL
}

\author{
A. B. SILVA, S. H. B. de FARIA e M. DeSOUZA \\ Universidade Estadual de Maringá, Departamento de Engenharia Química \\ e-mail para contato: batista.eq@gmail.com
}

\begin{abstract}
RESUMO - Muitas aplicações envolvem transferência de calor por convecção natural como o mecanismo principal de transferência de calor. Alguns exemplos são o resfriamento dos equipamentos eletrônicos como os transistores de potência e transferência de calor em serpentinas de refrigeração e linhas de transmissão. Neste trabalho utilizou-se o programa COMSOL Multiphysics para simular a convecção natural que ocorre quando duas placas paralelas, contendo um fluido entre elas, são mantidas a temperaturas diferentes. Esse processo envolve fenômenos simultâneos de transferência calor e de quantidade de movimento. Tal simultaneidade dá origem a um conjunto de equações diferenciais parciais não lineares. $\mathrm{O}$ objetivo do trabalho foi avaliar a influência que parâmetros como a densidade, a viscosidade, a condutividade térmica e a distância entre as placas exercem nos perfis de velocidade e de temperatura do fluido.
\end{abstract}

\section{INTRODUÇÃO}

A convecção natural de um fluido puro é um problema clássico de fenômeno de transporte que envolve simultaneamente transferência de calor e de quantidade de movimento. Esse fenômeno há muito vem sendo estudado, como em trabalhos de Batchelor (1954) e de Eckert e Carlson (1960), e mais recentemente em trabalhos de Liu et al. (2014) e de Roeleveld e Naylor (2014). Alguns exemplos de aplicação da convecção natural são o resfriamento dos equipamentos eletrônicos como os transistores de potência e transferência de calor em serpentinas de refrigeração e linhas de transmissão. No caso específico do escoamento devido à convecção natural entre duas placas paralelas a diferentes temperaturas, têm-se aplicações como o que ocorre com o fluido entre as placas de vidro de janelas de vidro duplo ou em paredes duplas de edifícios. Esse tipo de escoamento ocorre ainda na operação de colunas de Clusius-Dickel para separação de isótopos ou de soluções líquidas de substâncias orgânicas pelo efeito combinado da difusão térmica e da convecção natural. Um esquema para o caso de um fluido hipotético localizado entre duas placas paralelas é apresentado na Figura 1. Se as placas são mantidas a temperaturas diferentes, a porção do fluido em contato com a placa com temperatura maior, sofre um aumento de temperatura, o que diminui sua densidade, fazendo com que ele, agora mais leve, entre em um movimento convectivo ascendente. A porção do fluido em contato com a parede fria tem sua densidade aumentada e entra em movimento convectivo descendente. Tem-se então um sistema dinâmico em que há escoamento ascendente e descendente ao mesmo tempo. 


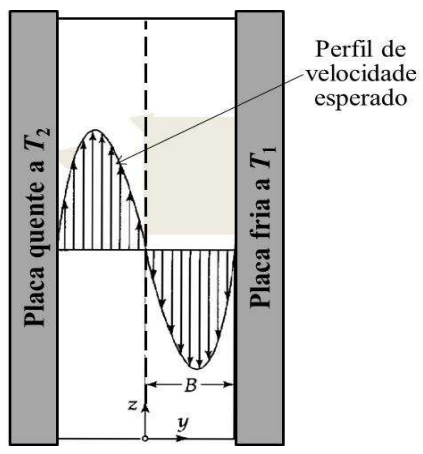

Figura 1 - Convecção natural laminar entre duas placas verticais com temperaturas diferentes.

Devido à condição de não deslizamento, a primeira camada de fluido diretamente em contato com a parede não se movimenta, com isso, a velocidade de escoamento na vertical é zero no ponto adjacente à parede e vai aumentando à medida que se distância da parede. Por outro lado, como a temperatura diferenciada da parede é a força motriz para o movimento do fluido, quanto mais longe da parede, menor é a força motriz, e, consequentemente menor a velocidade do fluido. Esses fatores combinados geram um perfil de velocidade parabólico para o escoamento. O mesmo ocorre na outra parede, contudo, a direção do escoamento é oposta. Assim, temos um perfil de velocidade parabólico direcionado para cima próximo à parede quente e um perfil de velocidade parabólico direcionado para baixo próximo à parede fria. Conforme as duas placas são aproximadas, os escoamentos adjacentes às placas influenciam um no outro, sendo que no ponto onde ocorre a mudança de sentido do escoamento a velocidade é zero. Tem-se então um perfil de velocidade total cúbico, composto pelos perfis de velocidade dos dois escoamentos, uma parábola voltada para cima e outra voltada para baixo, tendo valores de zero nas paredes e no ponto médio entre elas, conforme indicado na Figura 1. Bird et al. (2004), utilizando-se de diversas hipóteses simplificadoras, obtiveram uma solução analítica para o problema, a qual forneceu os seguintes perfis de velocidade e de temperatura:

$$
\begin{aligned}
& v=\left(\frac{\rho g \beta \Delta T B^{2}}{12 \mu}\right)\left[\left(\frac{y}{B}\right)^{3}-\left(\frac{y}{B}\right)\right] \\
& T=T_{0}+\frac{\left(T_{1}-T_{2}\right) y}{2 B}
\end{aligned}
$$

sendo $v$ a componente da velocidade do fluido na vertical, $T$ a temperatura do fluido, $\mu$ a viscosidade do fluido, $\rho$ a densidade do fluido na temperatura de referência $T_{0}=\left(T_{1}+T_{2}\right) / 2, T_{1}$ a temperatura da parede fria, $T_{2}$ a temperatura da parede quente, $g$ a aceleração da gravidade, $\beta$ o coeficiente de expansão térmica e $2 B$ a distância entre as placas. A Equação 1 indica um perfil cúbico de velocidade e a Equação 2 indica um perfil linear para a temperatura, ambos em relação à coordenada $y$. Neste trabalho, buscou-se simular numericamente o escoamento devido à convecção natural, verificando se as indicações do simulador seriam condizente com a teoria apresentada por 


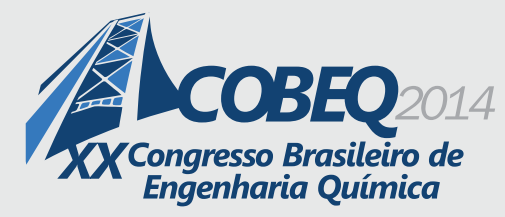

Bird et al. (2004). Após a verificação dessa etapa inicial, investigou-se a influência dos parâmetros: distância entre as placas, condutividade térmica, densidade e viscosidade do fluido sobre os perfis de velocidade e de temperatura do sistema.

\section{MODELAGEM MATEMÁTICA}

Para a modelagem matemática, considerou-se, neste trabalho, um subdomínio bidimensional formado por uma cavidade retangular com lados verticais de $10 \mathrm{~cm}$ e lados horizontais de $1 \mathrm{~cm}$, na qual se encontra um fluido puro em regime de escoamento laminar. A cavidade é formada por duas paredes verticais e duas placas horizontais isotérmicas, todas impermeáveis. Para que ocorresse convecção natural, a placa da esquerda foi mantida a uma temperatura maior que a temperatura da placa da direita.

O modelo matemático foi formulado levando-se em conta as seguintes considerações:

- devido às dimensões macroscópicas da cavidade, a condição de não deslizamento nas paredes pode ser utilizada;

- não há geração de calor na cavidade;

- as propriedades do fluido foram consideradas constantes, exceto a densidade no termo gravitacional nas equações de conservação de quantidade de movimento, que varia com a temperatura e segue a aproximação de Boussinesq;

- as propriedades do fluido foram avaliadas na temperatura de referência $T_{0}=\left(T_{1}+T_{2}\right) / 2$; e

- o fluido de trabalho é newtoniano.

O processo foi modelado para um escoamento incompressível (número de Mach $<0,3$ ) em regime laminar e em estado estacionário, com dissipação viscosa desprezível. Nessas condições as equações de Navier-Stokes puderam ser usadas em combinação com a equação de conservação de energia

$$
\begin{aligned}
& \frac{\partial u}{\partial y}+\frac{\partial v}{\partial z}=0 \\
& \rho\left(u \frac{\partial u}{\partial y}+v \frac{\partial u}{\partial z}\right)=-\frac{\partial p}{\partial y}+\mu\left(\frac{\partial^{2} u}{\partial y^{2}}+\frac{\partial^{2} u}{\partial z^{2}}\right) \\
& \rho\left(u \frac{\partial v}{\partial y}+v \frac{\partial v}{\partial z}\right)=-\frac{\partial p}{\partial z}+\mu\left(\frac{\partial^{2} v}{\partial y^{2}}+\frac{\partial^{2} v}{\partial z^{2}}\right)+\rho g \beta\left(T-T_{0}\right) \\
& u \frac{\partial T}{\partial y}+v \frac{\partial T}{\partial z}=\kappa\left(\frac{\partial^{2} T}{\partial y^{2}}+\frac{\partial^{2} T}{\partial z^{2}}\right)
\end{aligned}
$$

nas quais, $u$ e $v$ são as componentes da velocidade nas direções $y$ e $z$, respectivamente, $p$ é a pressão do fluido na cavidade e $\kappa$ é a condutividade térmica do fluido. A solução do modelo matemático, dado por esse sistema de equações diferenciais (Equações 3-6), foi obtida pelo método de elementos finitos com o auxílio do programa de simulação computacional COMSOL Multiphysics. 


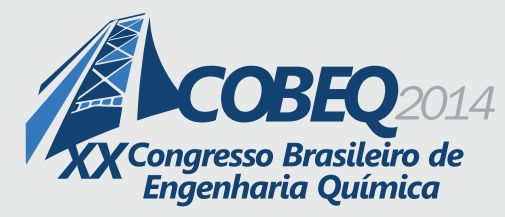

Inicialmente atribuiu-se para o fluido hipotético as mesmas propriedades do ar a $T_{0}=300 \mathrm{~K}$, sendo estas dispostas na Tabela 1. Todas as análises paramétricas efetuadas tiveram como ponto de partida as propriedades do fluido consideradas na Tabela 1 . Nas paredes verticais inseriu-se a condição de não deslizamento e as temperaturas diferentes em cada uma, sendo $5 \mathrm{~K}$ acima ou abaixo da temperatura inicial do sistema. Como valores iniciais, fez-se com que o sistema possuísse a temperatura média entre as temperaturas das paredes, pressão manométrica igual a zero e velocidade nula em qualquer direção.

Tabela 1 - Propriedades do fluido

\begin{tabular}{cll}
\hline Propriedade & \multicolumn{2}{c}{ Valor } \\
\hline$\rho$ & 1,1769 & $\mathrm{~kg} / \mathrm{m}^{3}$ \\
$\kappa$ & $2,6240 \times 10^{-2}$ & $\mathrm{~W} / \mathrm{m} . \mathrm{K}$ \\
$\mu$ & $1,8464 \times 10^{-5}$ & Pa.s \\
\hline
\end{tabular}

\section{RESULTADOS E DISCUSSÃO}

Para as condições iniciais, os perfis de temperatura e de velocidade obtidos são mostrados nas Figuras 2 e 3, respectivamente. Esses perfis indicam que o resultado da simulação numérica é condizente com a solução analítica proposta por Bird et al. (2004). Pois, obteve-se um perfil de temperatura linear, partindo de um máximo de $305 \mathrm{~K}$ na parede aquecida e indo até um mínimo de $295 \mathrm{~K}$ na parede fria. Para o perfil de velocidade, obteve-se um perfil cúbico, semelhante ao proposto por Bird et al. (2004). A velocidade máxima para essas condições foi de $0,0167 \mathrm{~m} / \mathrm{s}$.

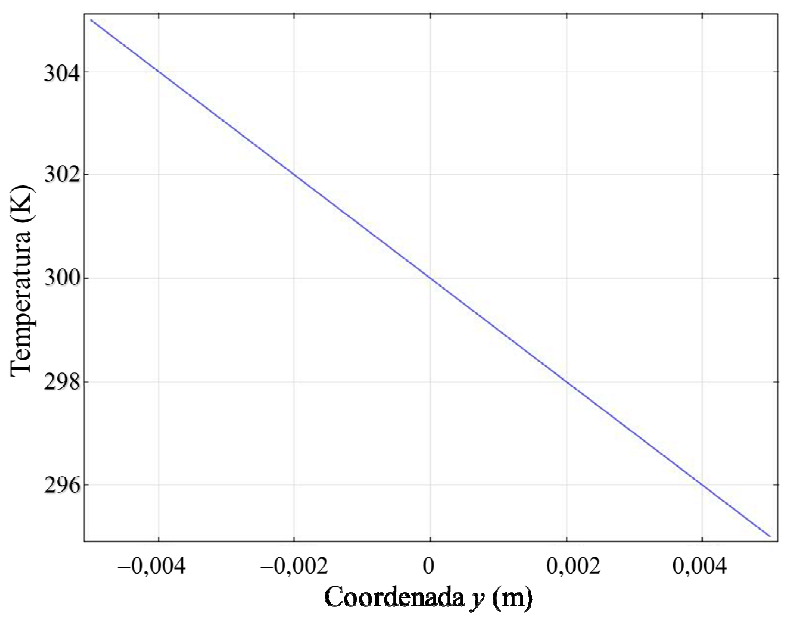

Figura 2 - Perfil de temperatura para fluidos com baixa densidade

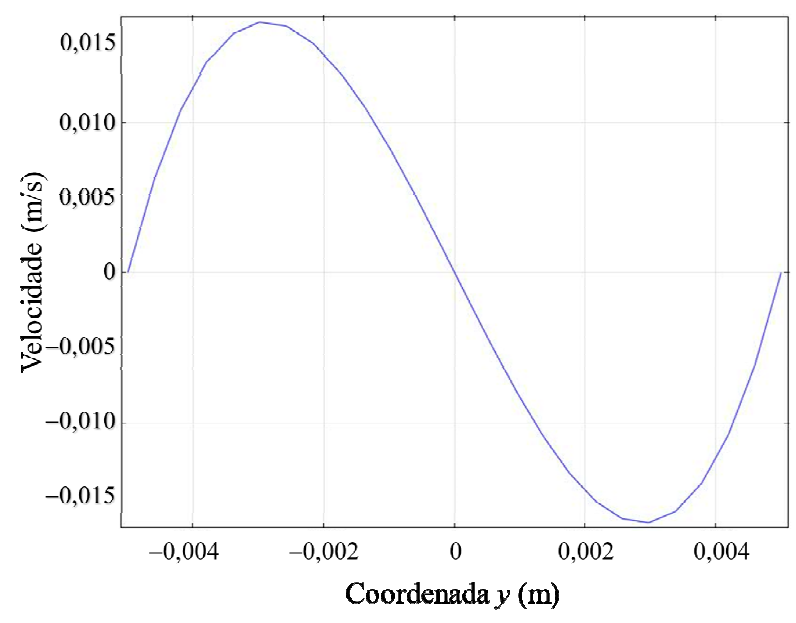

Figura 3 - Perfil de velocidade para fluidos com baixa densidade

\subsection{Variações na densidade}

Atribuindo-se ao fluido, a densidade de $1,7967 \mathrm{~kg} / \mathrm{m}^{3}$ (densidade igual à do $\mathrm{CO}_{2}$ a $300 \mathrm{~K}$ ) e 


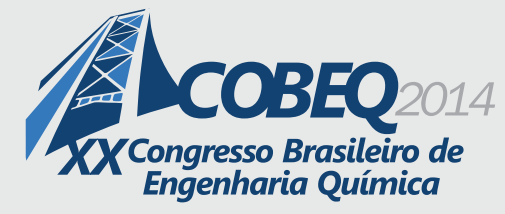

mantendo-se inalteradas as demais propriedades, obteve-se um perfil de temperatura igual ao anterior, contudo, o perfil de velocidade apresentou um máximo de $0,0255 \mathrm{~m} / \mathrm{s}$. O mesmo ocorreu para uma densidade de $0,0818 \mathrm{~kg} / \mathrm{m}^{3}$ (igual à do $\mathrm{H}_{2}$ a $300 \mathrm{~K}$ ), apresentando velocidade máxima de $0,00113 \mathrm{~m} / \mathrm{s}$. O valor máximo de densidade para o qual o modelo matemático proposto converge foi de $9,1 \mathrm{~kg} / \mathrm{m}^{3}$, valor esse quase oito vezes maior que a densidade do ar, porém ainda cerca de 100 vezes menor que a densidade da água líquida. Para essa densidade $\left(9,1 \mathrm{~kg} / \mathrm{m}^{3}\right)$ geraram-se os perfis de temperatura e velocidade apresentados nas Figuras 4 e 5, respectivamente.

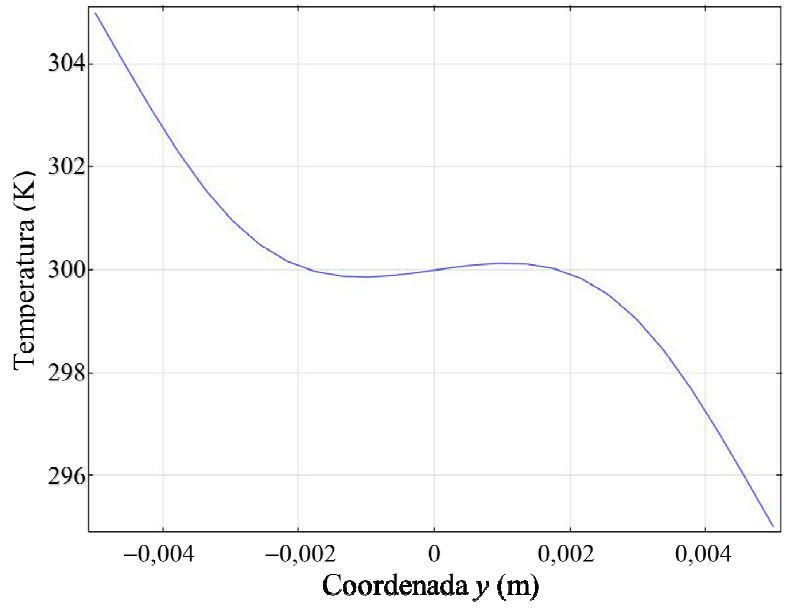

Figura 4 - Perfil de temperatura para fluidos com alta densidade

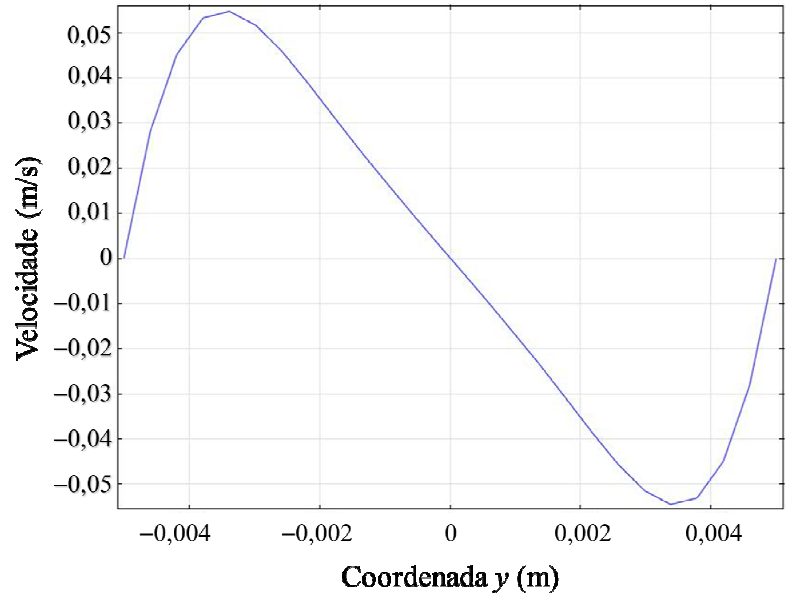

Figura 5 - Perfil de velocidade para fluidos com alta densidade

Os resultados apresentados na Tabela 2 indicam que a velocidade máxima do escoamento aumenta com o aumento da densidade. As Figuras 6 e 7 mostram a diferença global do escoamento no sistema. Para o fluido com maior densidade $\left(9,1 \mathrm{~kg} / \mathrm{m}^{3}\right)$ verifica-se uma menor velocidade nos pontos extremos do sistema, contudo, no centro do sistema o perfil de velocidade assume a forma cúbica já discutida, porém, para a relação de aspecto (altura/largura) do subdomínio modelado, a influência dos efeitos de borda não pode ser desprezada.

Tabela 2 - Características do perfil de temperatura e velocidade máxima do fluido em função da densidade do fluido

\begin{tabular}{ccc}
\hline Densidade $\left(\mathrm{kg} / \mathrm{m}^{3}\right)$ & Característica do perfil de temperatura & Velocidade máxima $(\mathrm{m} / \mathrm{s})$ \\
\hline 0,818 & Linear & 0,00113 \\
1,1769 & Linear & 0,01670 \\
1,7967 & Linear & 0,02550 \\
9,1000 & Altamente não linear & 0,05470 \\
\hline
\end{tabular}

\subsection{Variações na viscosidade}

Variando-se a viscosidade, diferentes valores de velocidade máxima foram encontrados, contudo, o perfil de temperaturas manteve-se linear e o de velocidades permaneceu cúbico tal como 
o da Figura 4. Os valores calculados encontram-se na Tabela 3. As viscosidade usadas foram $0,8963 \times 10^{-5}, 1,8464 \times 10^{-5}$ e $87,575 \times 10^{-5}$ (Pa.s), correspondentes às viscosidades, a $300 \mathrm{~K}$, do $\mathrm{H}_{2}$, do ar e da água líquida, respectivamente.

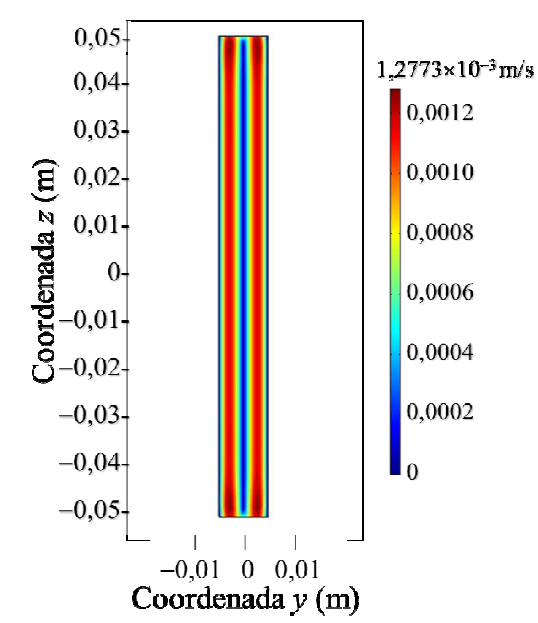

Figura 6 - Perfil de velocidade do fluido com menor densidade

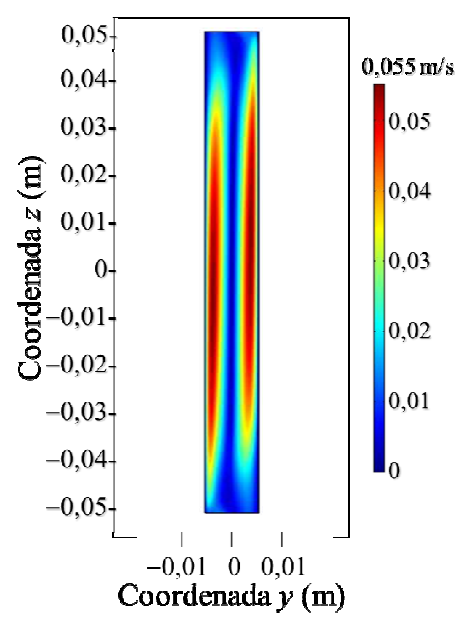

Figura 7 - Perfil de velocidade do fluido com maior densidade

Tabela 3 - Velocidade máxima de escoamento em função da viscosidade do fluido

\begin{tabular}{cc}
\hline Viscosidade $($ Pa.s) & Velocidade máxima $(\mathrm{m} / \mathrm{s})$ \\
\hline $0,8963 \times 10^{-5}$ & 0,03445 \\
$1,8464 \times 10^{-5}$ & 0,01670 \\
$87,575 \times 10^{-5}$ & 0,00352 \\
\hline
\end{tabular}

\subsection{Variações na condutividade térmica}

Os dados obtidos ao se atribuir ao fluido, diferentes valores de condutividade térmica, encontram-se dispostos na Tabela 4.

Tabela 4 - Características do perfil de temperatura e velocidade máxima de escoamento em função da condutividade térmica do fluido

\begin{tabular}{ccc}
\hline Condutividade $(\mathrm{W} / \mathrm{m} . \mathrm{K})$ & Perfil de temperatura & Velocidade máx. $(\mathrm{m} / \mathrm{s})$ \\
\hline $0,1820 \times 10^{-2}$ & Não linear & 0,0130 \\
$2,6240 \times 10^{-2}$ & Linear & 0,0167 \\
$60,960 \times 10^{-2}$ & Linear & 0,0167 \\
\hline
\end{tabular}

Uma explicação para esses resultados é que a diminuição da condutividade térmica aumenta a resistência à transferência de energia no fluido, reduzindo a influência da temperatura da parede e fazendo com que a densidade do fluido varie pouco no interior da cavidade (nas proximidades da parede), com isso, o movimento convectivo se torna mais lento. As temperaturas do sistema como 


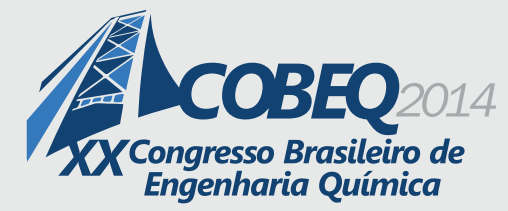

um todo são apresentadas nas Figuras 8 e 9.

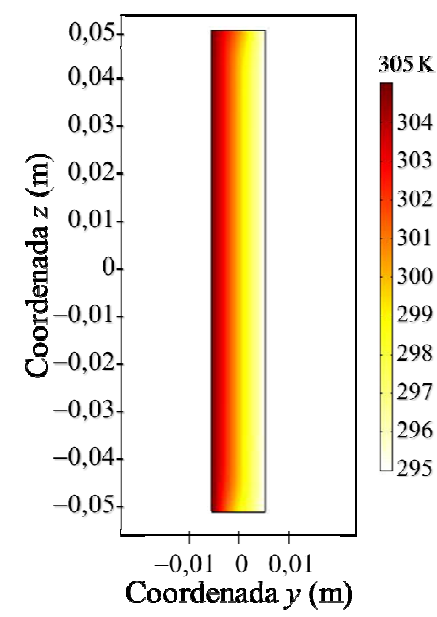

Figura 8 - Fluido com maior condutividade térmica
19 a 22 de outubro de 2014

Florianópolis/SC

\section{um todo são apresentadas nas Figuras 8 e 9.}


aumenta, o escoamento fica mais lento. Adicionalmente, para fluidos com densidade elevada e/ou com condutividade térmica baixa, a relação de aspecto altura/largura, deve ser maior para que os efeitos de borda possam ser desconsiderados.

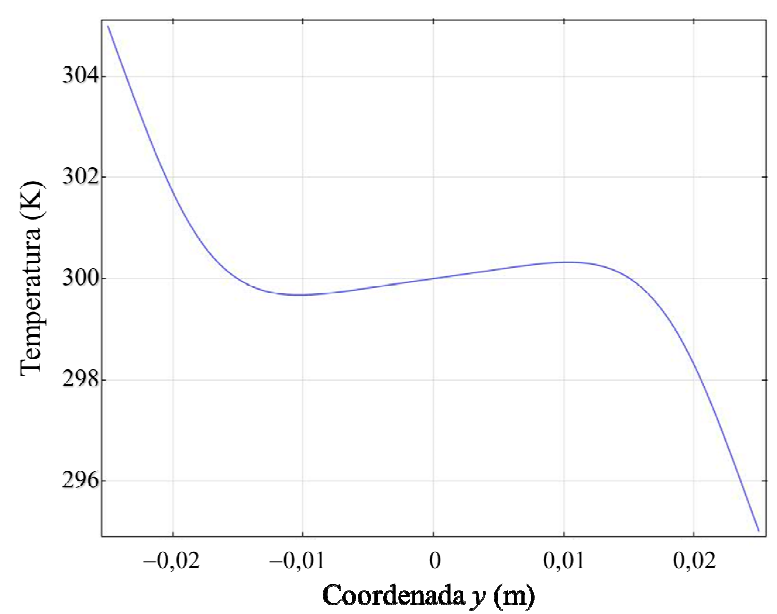

Figura 10 - Perfil de temperatura para o sistema com placas verticais mais espaçadas.

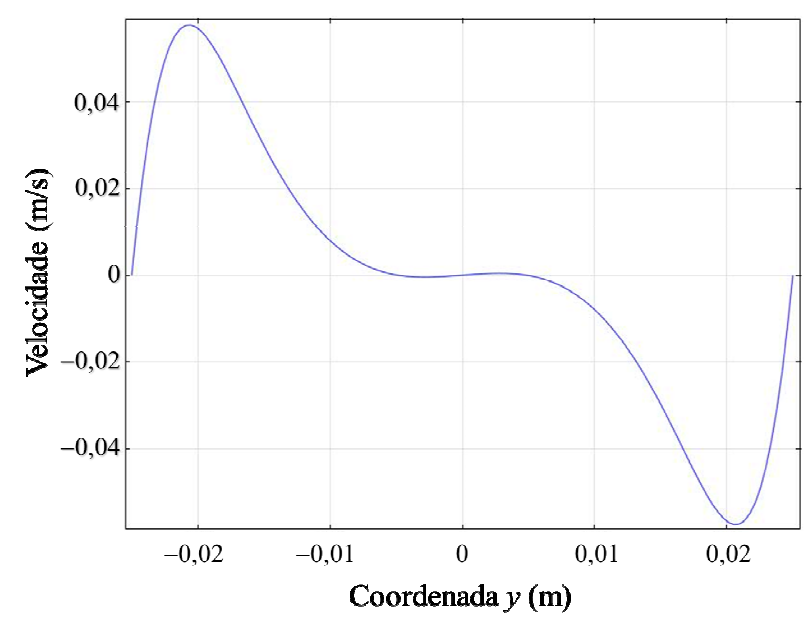

Figura 11 - Perfil de velocidade para o sistema com placas verticais mais espaçadas.

Tabela 5 - Características do perfil de temperatura e velocidade máxima de escoamento em função da distância entre as placas

\begin{tabular}{ccc}
\hline Distância $(\mathrm{cm})$ & Característica do perfil de temperaturas & Velocidade máxima $(\mathrm{m} / \mathrm{s})$ \\
\hline 0,1 & Linear & 0,000165 \\
1,0 & Linear & 0,016700 \\
2,0 & Não linear & 0,053000 \\
3,0 & Não linear & 0,057200 \\
5,0 & Altamente não linear & 0,057600 \\
\hline
\end{tabular}

\section{REFERÊNCIAS}

BATCHELOR, G. Heat transfer by free convection across a closed cavity between vertical bounderies at different temperatures. Quart. Appl. Math., v. 12, p. 209-233, 1954.

BIRD, R. B.; STEWART, W. E.; LIGHTFOOT, E. N. Fenômenos de Transporte. Rio de Janeiro: Editora LTC, 2004.

ECKERT, E. R. G.; CARLSON, W. O. Natural convection in an air layer enclosed between two vertical plates with different temperatures. Pergamon Press, v. 2, p. 106-120, 1961.

LIU, Y.; LEI, C.; PATTERSON, J. C. Natural Convection in a differentially heated cavity with two horizontal adiabatic fins on the siewalls. Int. J. Heat Mass Tran., v. 72, p. 23-36, 2014.

ROELEVELD, D.; NAYLOR, D. Flow visualization of natural convection in vertical channels with opposing buoyancy forces. Exp. Therm. Fluid Sci., v. 54, p. 61-70, 2014. 